$\mathrm{UG}-9 / 95$

\title{
DERIVATIVE EXPANSION FOR THE ONE-LOOP EFFECTIVE LAGRANGIAN IN QED
}

\author{
V.P. Gusynin ${ }^{1,2}$ and I.A.Shovkovy $y^{1,3}$ \\ 1 Bogolyubov Institute for Theoretical Physics \\ 252143, Kiev, Ukraine \\ 2 Institute for Theoretical Physics \\ University of Groningen, 9747 AG Groningen, The Netherlands \\ 3 Department of Applied Mathematics \\ University of Western Ontario, London, Canada N6A 5B7
}

\begin{abstract}
The derivative expansion of the one-loop effective Lagrangian in $\mathrm{QED}_{4}$ is considered. The first term in such an expansion is the famous Schwinger result for a constant electromagnetic field. In this paper we give an explicit expression for the next term containing two derivatives of the field strength $F_{\mu \nu}$. The results are presented for both fermion and scalar electrodynamics. Some possible applications of an inhomogeneous external field are pointed out.
\end{abstract}




\section{Introduction}

The effective Lagrangian for a constant electromagnetic field was calculated by Schwinger more than forty years ago [1]. The Schwinger Lagrangian has the virtue of being nonpertubative, taking into account the effects of the background field to all orders. However, its validity is restricted to constant fields and, since that time, much efforts have been devoted to finding a systematic way to generalize the result for strong slowly varying fields [2, 匂 or for the fields localized in a finite region of space [4]. Such a generalization would be of great interest from the physical point of view, since it can be applied to studying instabilities (such as spontaneous symmetry breakdown) in quantum field theory in external field [5, 6], or it can be used for the explanation of the narrow $e^{-} e^{+}$peaks observed in heavy-ion scattering experiments [7], where strong varying electromagnetic fields are indispensable ingredients. Also, nonperturbative information on the fermion determinant, closely connected with the one-loop effective action, is very important because fermion determinants produce an effective measure for gauge fields when the fermions are integrated out [8].

In the present paper, we calculate the low-energy effective action for fermions and bosons in $\mathrm{QED}_{4}$ as a derivative expansion around nonvanishing field strength

$$
\mathcal{L}_{e f f}=\mathcal{L}_{0}\left(F_{\mu \nu}\right)+\partial_{\lambda} F_{\alpha \beta} \partial_{\gamma} F_{\sigma \delta} \mathcal{L}_{1}^{\lambda \alpha \beta \gamma \sigma \delta}\left(F_{\mu \nu}\right)+\ldots,
$$

where $\mathcal{L}_{k}$ 's are some local functions of the field-strength tensor $F_{\mu \nu}$. In the derivative expansion (11) the first term $\mathcal{L}_{0}$ is the famous Schwinger result and we compute the next term $\mathcal{L}_{1}$.

Recently, an analogous calculation has been performed for QED in $2+1$ dimensions [3]. As for $3+1$ dimensions, we mention the paper [2] where the heat-kernel method was adopted to obtain the derivative expansion (1). However, it is impossible to compare our results with those in [2] because some important functions in the cited paper were not given explicitly. We comment more on comparison with results of Refs. [2, 3] at the end of the paper. 


\section{Derivative Expansion of the One-Loop Effec- tive Action in $\mathrm{QED}_{4}$}

The one-loop effective action in QED reduces to computing the fermion determinant

$$
\begin{aligned}
i W^{(1)}(A) & =\ln \operatorname{Det}(i \hat{\mathcal{D}}-m)=\frac{1}{2} \ln \operatorname{Det}\left(-\mathcal{D}_{\mu}^{2}+\frac{e}{2} \sigma_{\mu \nu} F^{\mu \nu}+m^{2}\right)= \\
& =\frac{1}{2} \int d^{4} x\left\langle x\left|\operatorname{tr} \ln \left(-\mathcal{D}_{\mu}^{2}+\frac{e}{2} \sigma_{\mu \nu} F^{\mu \nu}+m^{2}\right)\right| x\right\rangle .
\end{aligned}
$$

Here $\hat{\mathcal{D}}=\gamma^{\mu} \mathcal{D}_{\mu}$ and $\mathcal{D}_{\mu}=\partial_{\mu}+i e A_{\mu}$ is the covariant derivative, $\sigma_{\mu \nu}=i / 2\left[\gamma_{\mu}, \gamma_{\nu}\right]$ and $t r$ refers to spinor indices of Dirac matrices $\gamma_{\mu} ;|x\rangle$ are eigenstates for some self-conjugated operators $x_{\mu}$ of coordinates. Throughout the paper we use the Minkowski metric $\eta_{\mu \nu}=(1,-1,-1,-1)$.

With use of the identity $\ln A=-\int_{0}^{\infty} \exp (-i \tau A) d \tau / \tau$ for introducing the propertime coordinate, Eq.(2) can be represented through the diagonal matrix elements of the operator $U(\tau)=\exp (-i \tau H)$ :

$$
W^{(1)}(A)=\frac{i}{2} \int d^{4} x \int_{0}^{\infty} \frac{d \tau}{\tau} e^{-i m^{2} \tau} \operatorname{tr}\langle x|\exp (-i \tau H)| x\rangle,
$$

where the second order differential operator $H$ is given by

$$
H=\Pi_{\mu} \Pi^{\mu}+\frac{e}{2} \sigma_{\mu \nu} F^{\mu \nu}(x), \quad \Pi_{\mu}=-i \mathcal{D}_{\mu} .
$$

The evolution operator $U(\tau)$ satisfies the Schrödinger equation

$$
i \frac{d U(\tau)}{d \tau}=H U(\tau)
$$

together with the initial condition $U(0)=1$. In Euclidean space this operator is closely connected with the heat kernel operator. Because the matrix elements of $U(\tau)$ cannot be calculated for arbitrary external fields, several approximate regular schemes have been developed: the technique of asymptotic expansion at small values of the proper time $(\tau \rightarrow 0)$ [9-13], covariant perturbative theory [14, 15] and, at last, pseudodifferential operator technique [16-19]. The asymptotic expansion at $\tau \rightarrow 0$ is equivalent to the asymptotic expansion of the effective action in inverse powers of mass parameter $\mathrm{m}^{2}$. The coefficients of this expansion are the space-time integrals of local invariants, $E_{n}(x, x)(n=0,1,2, \ldots)$, of growing powers in field strenth $F_{\mu \nu}$ and its derivatives, called DeWitt-Seeley-Gilkey coefficients. The local (Schwinger-DeWitt) expansion is, therefore, an asymp-

totic approximation valid for weak and slowly varying background fields. The 
covariant perturbation theory of Barvinsky and Vilkovisky deals with the case of weak and rapidly varying fields. It corresponds to summing up all terms in the Schwinger-DeWitt series with a given power of field strength and any number of its derivatives. The covariant perturbation theory like the small proper-time expansion, fails in the case of large fields. An approximation scheme complimentary to the above mentioned ones would correspond to strong and slowly varying fields (derivative expansion). The lowest-order approximation of such a scheme is the case of covariantly constant background fields. In principle, the most general approach of pseudodifferential operators permits one to develop such a derivative expansion (see, for example, [20] and [2]), but in the present paper we shall follow another approach to derive the expansion (1).

The matrix elements $\langle x|\exp (-i \tau H)| x\rangle$ entering the right-hand side of Eq.(33) may be treated as the matrix elements of the evolution operator of a spinning particle, $\tau$ and $H$ being the proper time and Hamiltonian of the particle. The corresponding canonical momenta are $P_{\mu}$ 's which obey the commutation relations $\left[x_{\mu}, P^{\nu}\right]=i \delta_{\mu}^{\nu}$ and $\left\langle x\left|P_{\mu}\right| y\right\rangle=-i \partial_{\mu} \delta(x-y)$. Following the standard method [21], the transition amplitude $\langle z|U(\tau)| y\rangle$ between points $x(0)=y$ and $x(\tau)=z$ can be written in terms of a path integral over c-number $\left(x_{\mu}(t)\right)$ and Grassmann $\left(\psi_{\mu}(t)\right)$ coordinates:

$$
\operatorname{tr}\langle z|U(\tau)| y\rangle=N^{-1} \int \mathcal{D}[x(t), \psi(t)] \exp \left\{i \int_{0}^{\tau} d t\left[L_{\text {bos }}(x(t))+L_{f e r}(\psi(t), x(t))\right]\right\},
$$

where $N$ is a normalization factor, and

$$
\begin{gathered}
L_{b o s}(x)=-\frac{1}{4} \frac{d x_{\nu}}{d t} \frac{d x^{\nu}}{d t}-e A_{\nu}(x) \frac{d x^{\nu}}{d t}, \\
L_{f e r}(\psi, x)=\frac{i}{2} \psi_{\nu} \frac{d \psi^{\nu}}{d t}-i e \psi^{\nu} \psi^{\lambda} F_{\nu \lambda}(x) .
\end{gathered}
$$

The integration in Eq.(6i) goes over trajectories $x^{\mu}(t)$ and $\psi^{\mu}(t)$ parametrized by $t \in[0, \tau]$ and obeying the boudary conditions

$$
x(0)=y, \quad x(\tau)=z, \quad \psi(0)=-\psi(\tau) .
$$

The Lagrangian $L_{b o s}(x)+L_{f e r}(\psi, x)$ may be treated as Lagrangian of the relativistic spinning particle in external electromagnetic field [21, 22, 231. 't The first-quantized representation for the effective action, given by Eqs.(3), (6)-(8),

\footnotetext{
${ }^{1}$ For a more sophisticated representation in terms of path-integral with reparametrizationinvariant Lagrangian, see [21, 23]. We note that from the point of view of further using the first quantized path integral in applications it would be quite desirable to have a representation for the evolution operator in terms of boson operators only. Since $\gamma$-matrices enter the operator (14) through $\sigma_{\mu \nu}$, satisfying $S O(3,1)$ Lie algebra, such a representation should exist.
} 
can be used as a starting point for developing approximate schemes for computing the expansion in the case of arbitrary external fields. The path integral (6) is the particle theory analogue of the Polyakov path integral in string theory, and motivated by this fact, Strassler [24] has developed the first-quantized perturbation theory for calculating the one-loop effective action. Moreover, such an approach to ordinary field theory, based on path integrals defined on one-dimensional world lines, can be extended to the evaluation of Feynman diagrams for Green's functions at arbitrary loop order 25] and to the computation of form-factors [26]. In the last case it was shown how the higher derivative terms appearing in the effective action can be effectively summed up and lead to the results obtained in the heat kernel approach of Barvinsky and Vilkovisky [14].

In order to generate the expansion (11) from the path integral (3) it is convenient to choose a special gauge condition for the potential $A_{\mu}(x)$, namely the FockSchwinger gauge [27]

$$
\left(x^{\nu}-y^{\nu}\right) A_{\nu}(x)=0,
$$

which leads to the series

$$
\begin{aligned}
A_{\nu}(x) & =\frac{1}{2}\left(x^{\lambda}-y^{\lambda}\right) F_{\lambda \nu}(y)+\frac{1}{3}\left(x^{\lambda}-y^{\lambda}\right)\left(x^{\sigma}-y^{\sigma}\right) \partial_{\sigma} F_{\lambda \nu}(y) \\
& +\frac{1}{8}\left(x^{\lambda}-y^{\lambda}\right)\left(x^{\sigma}-y^{\sigma}\right)\left(x^{\mu}-y^{\mu}\right) \partial_{\sigma} \partial_{\mu} F_{\lambda \nu}(y)+\ldots \\
& =\sum_{n=0}^{\infty} \frac{\left(x^{\lambda}-y^{\lambda}\right)\left(x^{\nu_{1}}-y^{\nu_{1}}\right) \ldots\left(x^{\nu_{n}}-y^{\nu_{n}}\right)}{n !(n+2)} \partial_{\nu_{1}} \partial_{\nu_{2}} \ldots \partial_{\nu_{n}} F_{\lambda \nu}(y)
\end{aligned}
$$

Carring out the change of the variable $x(t)$ for $x^{\prime}(t)=x(t)-y$ in the path integral (6) (henceforth we omit the prime) and substituting (11) into (6), we obtain

$$
\begin{aligned}
& \operatorname{tr}\langle z|U(\tau)| y\rangle=N^{-1} \int D[x(t), \psi(t)] \exp \left[i \int _ { 0 } ^ { \tau } d t \left(-\frac{1}{4} \frac{d x_{\nu}}{d t} \frac{d x^{\nu}}{d t}-\frac{e}{2} x^{\lambda} F_{\lambda \nu}(y)\right.\right. \\
& \left.\left.\times \frac{d x^{\nu}}{d t}+L_{2}(x)\right)\right] \exp \left[i \int_{0}^{\tau} d t\left(\frac{i}{2} \psi_{\nu} \frac{d \psi^{\nu}}{d t}-i e \psi^{\nu} \psi^{\lambda} F_{\nu \lambda}(y)+L_{3}(x, \psi)\right)\right]
\end{aligned}
$$

The new boundary conditions for $x(t)$ are $x(0)=0$ and $x(\tau)=z-y$. In Eq.(12) $F_{\mu \nu}$ does not depend on $x(t)$. The expressions for the interacting terms, $L_{2}(x)$ and $L_{3}(x, \psi)$, containing derivatives of $F_{\mu \nu}$ with respect to coordinates take the form:

$$
\begin{aligned}
L_{2}(x) & =\sum_{n=1}^{\infty} \frac{e F_{\nu_{0} \nu_{1}, \nu_{2} \ldots \nu_{n+1}}}{n !(n+2)} \frac{d x^{\nu_{0}}}{d t} x^{\nu_{1}}(t) \ldots x^{\nu_{n+1}}(t) \\
& =\frac{e}{3} F_{\nu \lambda, \sigma} \frac{d x^{\nu}}{d t} x^{\lambda} x^{\sigma}+\frac{e}{8} F_{\nu \lambda, \sigma \kappa} \frac{d x^{\nu}}{d t} x^{\lambda} x^{\sigma} x^{\kappa}+\ldots
\end{aligned}
$$




$$
\begin{aligned}
L_{3}(x, \psi) & =-\sum_{n=1}^{\infty} \frac{i}{n !} e F_{\lambda \mu, \nu_{1} \ldots \nu_{n}} \psi^{\lambda}(t) \psi^{\mu}(t) x^{\nu_{1}}(t) \ldots x^{\nu_{n}}(t) \\
& =-i e F_{\nu \lambda, \sigma} \psi^{\nu} \psi^{\lambda} x^{\sigma}-\frac{i e}{2} F_{\nu \lambda, \sigma \kappa} \psi^{\nu} \psi^{\lambda} x^{\sigma} x^{\kappa}+\ldots
\end{aligned}
$$

Here we use a conventional notation for derivatives: $F_{\lambda \mu, \nu_{1} \nu_{2} \ldots \nu_{n}}(x)=$ $\partial_{\nu_{1}} \partial_{\nu_{2}} \ldots \partial_{\nu_{n}} F_{\lambda \mu}(x)$

Introducing c-number and Grassmann external sources, we rewrite (12) as

$$
\begin{aligned}
\operatorname{tr}\langle z|U(\tau)| y\rangle & =\exp \left[i \int_{0}^{\tau} d t\left(L_{2}\left(\frac{1}{i} \frac{\delta}{\delta \eta(t)}\right)+L_{3}\left(\frac{1}{i} \frac{\delta}{\delta \eta(t)},-\frac{\delta}{\delta \xi(t)}\right)\right)\right] \\
& \times\left. Z_{\tau}[\eta, \xi](z ; y)\right|_{\eta=0, \xi=0},
\end{aligned}
$$

where the generating functional is defined by

$$
\begin{aligned}
& Z_{\tau}[\eta, \xi](z ; y)=N^{-1} \int D[x(t), \psi(t)] \exp \left[\frac { i } { 2 } \int _ { 0 } ^ { \tau } d t \left(-\frac{1}{2} \frac{d x_{\nu}}{d t} \frac{d x^{\nu}}{d t}-e x^{\lambda} F_{\lambda \nu}(y)\right.\right. \\
& \left.\left.\times \frac{d x^{\nu}}{d t}+2 \eta_{\nu} x^{\nu}\right)\right] \exp \left[-\frac{1}{2} \int_{0}^{\tau} d t\left(\psi_{\nu} \frac{d \psi^{\nu}}{d t}-2 e \psi^{\nu} \psi^{\lambda} F_{\nu \lambda}(y)+2 \xi_{\nu} \psi^{\nu}\right)\right]
\end{aligned}
$$

Since the expression in the exponent of the right-hand side of (16) is quadratic in the variables $x$ and $\psi$, the calculation of the generating functional $Z_{\tau}[\eta, \xi](z ; y)$ can be reduced to the calculation of the determinants of the following onedimensional differential operators:

$$
O_{1}=\frac{\eta_{\mu \nu}}{2} \frac{d^{2}}{d t^{2}}-e F_{\mu \nu} \frac{d}{d t} \quad \text { and } \quad O_{2}=i \eta_{\mu \nu} \frac{d}{d t}-2 i e F_{\mu \nu}
$$

defined on the interval $[0, \tau]$ with the periodic and antiperiodic boudary conditions, respectively. Taking into account the initial condition

$$
Z_{\tau=0}[\eta, \xi](z ; y)=\delta(z-y)
$$

which is equivalent to the operator equality $U(0)=1$, and introducing two independent invariants constructed from the field tensor $F_{\mu \nu}$ :

$$
K_{+}=\sqrt{\sqrt{\mathcal{F}^{2}+\mathcal{G}^{2}}+\mathcal{F}} \quad \text { and } \quad K_{-}=\sqrt{\sqrt{\mathcal{F}^{2}+\mathcal{G}^{2}}-\mathcal{F}}
$$

with

$$
\mathcal{F}=-\frac{1}{4} F^{\mu \nu} F_{\mu \nu} \quad \text { and } \quad \mathcal{G}=\frac{1}{4} \epsilon^{\mu \nu \lambda \kappa} F_{\lambda \kappa} F_{\mu \nu}
$$


one can represent the result of the integration in (16) at coincident arguments $z=y=x$ as

$$
\begin{aligned}
Z_{\tau}[\eta, \xi](x ; x)= & -\frac{i}{4 \pi^{2} \tau^{2}}\left(e \tau K_{+}\right)\left(e \tau K_{-}\right) \operatorname{coth}\left(e \tau K_{+}\right) \cot \left(e \tau K_{-}\right) \\
& \cdot \exp \left(\frac{i}{2} S_{c l}^{b o s}[\eta]-\frac{1}{2} S_{c l}^{f e r}[\xi]\right)
\end{aligned}
$$

where the quadratic in external sourses forms, $S_{c l}^{b o s}$ and $S_{c l}^{f e r}$, are defined by

$$
\begin{aligned}
S_{c l}^{b o s}[\eta] & =\int_{0}^{\tau} d t_{1} \int_{0}^{\tau} d t_{2} \eta_{\nu}\left(t_{1}\right) D^{\nu \lambda}\left(t_{1}, t_{2}\right) \eta_{\lambda}\left(t_{2}\right), \\
S_{c l}^{f e r}[\xi] & =\int_{0}^{\tau} d t_{1} \int_{0}^{\tau} d t_{2} \xi_{\nu}\left(t_{1}\right) S^{\nu \lambda}\left(t_{1}, t_{2}\right) \xi_{\lambda}\left(t_{2}\right),
\end{aligned}
$$

The Green functions entering the last expression are defined by the formulae:

$$
\begin{aligned}
D^{\nu \lambda}\left(t_{1}, t_{2}\right) & =\sum_{j=1}^{4} A_{(j)}^{\nu \lambda} \frac{1}{2 e f_{j}}\left(\epsilon\left(t_{1}-t_{2}\right)\left(1-\exp \left[2 e f_{j}\left(t_{1}-t_{2}\right)\right]\right)\right. \\
& +\operatorname{coth}\left(e f_{j} \tau\right)\left(1+\exp \left[2 e f_{j}\left(t_{1}-t_{2}\right)\right]\right) \\
& \left.-\sinh ^{-1}\left(e f_{j} \tau\right)\left(\exp \left[e f_{j}\left(\tau-2 t_{2}\right)\right]+\exp \left[e f_{j}\left(2 t_{1}-\tau\right)\right]\right)\right), \\
S^{\nu \lambda}\left(t_{1}, t_{2}\right)= & \sum_{j=1}^{4} A_{(j)}^{\nu \lambda} \frac{1}{2}\left(\epsilon\left(t_{1}-t_{2}\right)-\tanh \left(e f_{j} \tau\right)\right) \exp \left[2 e f_{j}\left(t_{1}-t_{2}\right)\right] .
\end{aligned}
$$

Here we use the matrices $A_{(j)}^{\nu \lambda}$ which were originaly introduced in [28]. The explicit expressions for them are

$$
A_{\mu \nu(j)}=\frac{-\bar{f}_{j}^{2} g_{\mu \nu}+f_{j} F_{\mu \nu}+F_{\mu \nu}^{2}-i \bar{f}_{j} \stackrel{F}{F}_{\mu \nu}}{2\left(f_{j}^{2}-\bar{f}_{j}^{2}\right)}
$$

where

$$
\begin{gathered}
f_{1}=i K_{-}, f_{2}=-i K_{-} \quad, \quad f_{3}=K_{+}, f_{4}=-K_{+} \\
\bar{f}_{1}=-K_{+}, \overline{f_{2}}=K_{+} \quad, \quad \overline{f_{3}}=-i K_{-}, \bar{f}_{4}=i K_{-} .
\end{gathered}
$$

The main property of matrices (26) that was used in (24) and (25) is

$$
F^{\nu \lambda} A_{(i) \lambda \mu}=A_{(i)}^{\nu \kappa} F_{\kappa \mu}=f_{i} A_{(i) \mu}^{\nu} .
$$

Other useful properties of these matrices that will be used below are:

$$
\sum_{i=1}^{4} A_{(i)}^{\mu \nu}=\eta^{\mu \nu}, \quad A_{\mu(i)}^{\mu}=1, \quad A_{(i)}^{\mu \nu} A_{\nu \lambda(j)}=\delta_{i j} A_{\lambda(j)}^{\mu} .
$$


As can be easily seen, in the case of vanishing field the propagators $D^{\nu \lambda}\left(t_{1}, t_{2}\right)$ and $S\left(t_{1}, t_{2}\right)$ are reduced to the free Green functions used in [26].

Substitution of (21) and (24), (25) into (15) leads to the expression for $\operatorname{tr}\langle x|U| x\rangle$. The last step is to expand the exponent in terms of derivatives and to keep only two-derivative terms. Therefore, one obtains:

$$
\begin{gathered}
\operatorname{tr}\langle x|U(\tau)| x\rangle=\left(1+i \int_{0}^{\tau} d t\left[\mathbf{V}_{2}(t)+\mathbf{W}_{2}(t)\right]-\frac{1}{2} \int_{0}^{\tau} \int_{0}^{\tau} d t_{1} d t_{2}\left[\mathbf{V}_{1}\left(t_{1}\right) \mathbf{V}_{1}\left(t_{2}\right)\right.\right. \\
\left.\left.+\mathbf{W}_{1}\left(t_{1}\right) \mathbf{W}_{1}\left(t_{2}\right)\right]-\int_{0}^{\tau} \int_{0}^{\tau} d t_{1} d t_{2} \mathbf{V}_{1}\left(t_{1}\right) \mathbf{W}_{1}\left(t_{2}\right)\right)\left.Z_{\tau}[\eta, \xi](x, x)\right|_{\eta=0, \xi=0}, \quad,
\end{gathered}
$$

where

$$
\begin{aligned}
\mathbf{V}_{1}(t) & =\frac{i}{3} e F_{\nu \lambda, \mu} \lim _{t_{0} \rightarrow t} \frac{d}{d t_{0}} \frac{\delta^{3}}{\delta \eta_{\nu}\left(t_{0}\right) \delta \eta_{\lambda}(t) \delta \eta_{\mu}(t)} \\
\mathbf{V}_{2}(t) & =\frac{1}{8} e F_{\nu \lambda, \mu \kappa} \lim _{t_{0} \rightarrow t} \frac{d}{d t_{0}} \frac{\delta^{4}}{\delta \eta_{\nu}\left(t_{0}\right) \delta \eta_{\lambda}(t) \delta \eta_{\mu}(t) \delta \eta_{\kappa}(t)} \\
\mathbf{W}_{1}(t) & =-e F_{\nu \lambda, \mu} \frac{\delta^{2}}{\delta \xi_{\nu}(t) \delta \xi_{\lambda}(t)} \frac{\delta}{\delta \eta_{\mu}(t)} \\
\mathbf{W}_{2}(t) & =\frac{i}{2} e F_{\nu \lambda, \mu \kappa} \frac{\delta^{2}}{\delta \xi_{\nu}(t) \delta \xi_{\lambda}(t)} \frac{\delta^{2}}{\delta \eta_{\mu}(t) \delta \eta_{\kappa}(t)}
\end{aligned}
$$

Substituting the generating function (21) which depends on the Green functions (24) and (25), one can rewrite (29) in the form:

$$
\begin{aligned}
& \operatorname{tr}\langle x|U(\tau)| x\rangle=-\frac{i\left(e \tau K_{-}\right)\left(e \tau K_{+}\right) \cot \left(e \tau K_{-}\right) \operatorname{coth}\left(e \tau K_{+}\right)}{4 \pi^{2} \tau^{2}}\left[1-\frac{i}{8} e F_{\nu \lambda, \mu \kappa}\right. \\
\times & \int_{0}^{\tau} d t\left(\dot{D}^{\nu \lambda}(t, t) D^{\mu \kappa}(t, t)+\dot{D}^{\nu \mu}(t, t) D^{\lambda \kappa}(t, t)+\dot{D}^{\nu \kappa}(t, t) D^{\lambda \mu}(t, t)+4 S^{\nu \lambda}(t, t)\right. \\
\times & \left.D^{\mu \kappa}(t, t)\right)-\frac{i}{18} e^{2} F_{\nu \lambda, \mu} F_{\sigma \kappa, \rho} \int_{0}^{\tau} \int_{0}^{\tau} d t_{1} d t_{2}\left(9 D ^ { \mu \rho } ( 1 , 2 ) \left(S^{\kappa \sigma}(2,2) S^{\lambda \nu}(1,1)\right.\right. \\
- & \left.2 S^{\kappa \lambda}(2,1) S^{\sigma \nu}(2,1)\right)+6 S^{\sigma \kappa}(2,2)\left(\dot{D}^{\nu \lambda}(1,1) D^{\mu \rho}(1,2)+\dot{D}^{\nu \mu}(1,1) D^{\lambda \rho}(1,2)\right. \\
+ & \left.\dot{D}^{\nu \rho}(1,2) D^{\lambda \mu}(1,1)\right)+\dot{D}^{\nu \lambda}(1,1) \dot{D}^{\sigma \kappa}(2,2) D^{\mu \rho}(1,2)+2 \dot{D}^{\nu \lambda}(1,1)\left(\dot{D}^{\sigma \rho}(2,2)\right. \\
\times & \left.D^{\mu \kappa}(1,2)+\dot{D}^{\sigma \mu}(2,1) D^{\kappa \rho}(2,2)\right)+\dot{D}^{\nu \mu}(1,1) \dot{D}^{\sigma \rho}(2,2) D^{\lambda \kappa}(1,2)+2 \dot{D}^{\nu \kappa}(1,2) \\
\times & \left(\dot{D}^{\sigma \rho}(2,2) D^{\lambda \mu}(1,1)+\dot{D}^{\sigma \mu}(2,1) D^{\lambda \rho}(1,2)\right)+\dot{D}^{\nu \kappa}(1,2) \dot{D}^{\sigma \lambda}(2,1) D^{\mu \rho}(1,2) \\
+ & \dot{D}^{\nu \rho}(1,2) \dot{D}^{\sigma \mu}(2,1) D^{\lambda \kappa}(1,2)+\ddot{D}^{\nu \sigma}(1,2)\left(D^{\lambda \mu}(1,1) D^{\kappa \rho}(2,2)+D^{\lambda \kappa}(1,2)\right.
\end{aligned}
$$




$$
\left.\left.\left.\times D^{\mu \rho}(1,2)+D^{\lambda \rho}(1,2) D^{\mu \kappa}(1,2)\right)\right)\right] .
$$

Here the dotted functions are defined by

$$
\begin{aligned}
& \dot{D}^{\mu \nu}(1,2) \stackrel{\text { def }}{=} \frac{\partial}{\partial t_{1}} D^{\mu \nu}\left(t_{1}, t_{2}\right), \\
& \ddot{D}^{\mu \nu}(1,2) \stackrel{\text { def }}{=} \frac{\partial^{2}}{\partial t_{1} \partial t_{2}} D^{\mu \nu}\left(t_{1}, t_{2}\right), \\
& \dot{D}^{\mu \nu}(t, t) \stackrel{\text { def }}{=} \lim _{t_{0} \rightarrow t} \frac{\partial}{\partial t_{0}} D^{\mu \nu}\left(t_{0}, t\right) .
\end{aligned}
$$

The straightforward, though tedious, computation gives the result

$$
\begin{aligned}
& \operatorname{tr}\langle x|U(\tau)| x\rangle=-\frac{i}{4 \pi^{2} \tau^{2}}\left(e \tau K_{-}\right)\left(e \tau K_{+}\right) \cot \left(e \tau K_{-}\right) \operatorname{coth}\left(e \tau K_{+}\right) \\
\times & {\left[1-\frac{i}{8} e F_{\nu \lambda, \mu \kappa} \sum_{j, l}\left(C^{V}\left(f_{j}, f_{l}\right)\left(A_{(j)}^{\nu \lambda} A_{(l)}^{\mu \kappa}+2 A_{(j)}^{\nu \mu} A_{(l)}^{\lambda \kappa}\right)+2 C^{W}\left(f_{j}, f_{l}\right) A_{(j)}^{\lambda \nu} A_{(l)}^{\mu \kappa}\right)\right.} \\
- & \frac{i}{18} e^{2} F_{\nu \lambda, \mu} F_{\sigma \kappa, \rho} \sum_{j, l, k}\left(9 C_{1}^{W W}\left(f_{j}, f_{l}, f_{k}\right) A_{(j)}^{\kappa \sigma} A_{(l)}^{\lambda \nu} A_{(k)}^{\mu \rho}+9 C_{2}^{W W}\left(f_{j}, f_{l}, f_{k}\right) A_{(j)}^{\kappa \lambda} A_{(l)}^{\sigma \nu}\right. \\
\times & A_{(k)}^{\mu \rho}+6 C_{1}^{V W}\left(f_{j}, f_{l}, f_{k}\right) A_{(j)}^{\sigma \kappa}\left(A_{(l)}^{\nu \lambda} A_{(k)}^{\mu \rho}+A_{(l)}^{\nu \mu} A_{(k)}^{\lambda \rho}\right)+6 C_{2}^{V W}\left(f_{j}, f_{l}, f_{k}\right) A_{(j)}^{\sigma \kappa} A_{(l)}^{\nu \rho} A_{(k)}^{\lambda \mu} \\
- & C_{1}^{V V}\left(f_{j}, f_{l}, f_{k}\right)\left(A_{(j)}^{\nu \lambda} A_{(l)}^{\kappa \sigma} A_{(k)}^{\mu \rho}+A_{(j)}^{\nu \mu} A_{(l)}^{\kappa \rho} A_{(k)}^{\lambda \sigma}+2 A_{(j)}^{\nu \lambda} A_{(l)}^{\kappa \rho} A_{(k)}^{\mu \sigma}\right) \\
- & C_{2}^{V V}\left(f_{j}, f_{l}, f_{k}\right)\left(A_{(j)}^{\nu \sigma} A_{(l)}^{\kappa \lambda} A_{(k)}^{\mu \rho}+A_{(j)}^{\nu \rho} A_{(l)}^{\kappa \mu} A_{(k)}^{\lambda \sigma}+2 A_{(j)}^{\nu \sigma} A_{(l)}^{\kappa \mu} A_{(k)}^{\lambda \rho}\right) \\
- & 2 C_{3}^{V V}\left(f_{j}, f_{l}, f_{k}\right)\left(A_{(j)}^{\nu \lambda} A_{(l)}^{\kappa \mu} A_{(k)}^{\sigma \rho}+A_{(j)}^{\kappa \rho} A_{(l)}^{\nu \sigma} A_{(k)}^{\lambda \mu}\right)-C_{4}^{V V}\left(f_{j}, f_{l}, f_{k}\right) A_{(j)}^{\nu \kappa} A_{(l)}^{\lambda \mu} A_{(k)}^{\sigma \rho} \\
- & \left.\left.C_{5}^{V V}\left(f_{j}, f_{l}, f_{k}\right) A_{(j)}^{\nu \kappa}\left(A_{(l)}^{\lambda \sigma} A_{(k)}^{\mu \rho}+A_{(l)}^{\lambda \rho} A_{(k)}^{\mu \sigma}\right)\right)\right],
\end{aligned}
$$

where the coefficients $C_{i}^{X Y}(\alpha, \beta, \gamma)$ (where $\left.X, Y \in\{V, W\}\right)$ are given in Appendix.

In principle, the expression (35) is our final result. It remains only to substitute the last expression into (3) and to renormalize field and charge according to the rule

$$
\begin{gathered}
\left(e^{p h y s}\right)^{2}=\frac{e^{2}}{1+\mathbf{C} e^{2}}, \quad A_{\mu}^{p h y s}=\sqrt{1+\mathbf{C} e^{2}} A_{\mu} \\
\mathbf{C}=\frac{e^{2}}{12 \pi} \int_{0}^{\infty} \frac{d \tau}{\tau} \exp \left(-i m^{2} \tau\right) .
\end{gathered}
$$

Note that the renormalization used merely means performing some subtraction (precisely the same as in the origional Schwinger paper [1]) from the term containing no derivatives of field strength and changing all bare quantities for physical ones. 
As was mentioned at the begining of the paper, the derivative expansion in $\mathrm{QED}_{4}$ was also studied in 22]. The result of that paper, however, was presented in an explicit form only for the special case of the electromagnetic field:

$$
\mathcal{G}=0, \quad F^{\mu \nu}(x)=\Phi(x) \mathbf{F}^{\mu \nu}
$$

where $\Phi(x)$ is a scalar function and $\mathbf{F}^{\mu \nu}$ is a constant matrix. Under the particular choice of the field configuration given by (38) the derivative part of the diagonal matrix elements (35) reads

$$
\operatorname{tr}\langle x|U(\tau)| x\rangle_{d e r}=\frac{1}{(4 \pi)^{2} \tau} \frac{\partial_{\mu} \Phi \partial^{\mu} \Phi}{\Phi^{2}}\left(3 \omega^{2} Y^{4}-3 \omega Y^{3}-4 \omega^{2} Y^{2}+3 \omega Y+\omega^{2}\right),
$$

where $\omega=e \tau \sqrt{2 \mathcal{F}}, Y=\operatorname{coth}(\omega)$. We note that this result was obtained in [2] by means of symbolic calculations on a computer using the REDUCE package.

Now turning to the calculation of the derivative expansion for the scalar electrodynamics, one does not need to repeat all the calculations similar to those made above. In order to see this, we recall that the effective one-loop Lagrangian in this case reads

$$
L_{b o s}^{(1)}(x)=-i \int_{0}^{\infty} \frac{d \tau}{\tau}\left\langle x\left|U_{b o s}(\tau)\right| x\right\rangle e^{-i m^{2} \tau} .
$$

The evolution connected with the transition amplitude, $\left\langle z\left|U_{b o s}(\tau)\right| y\right\rangle$, is described now by the Hamiltonian (compare with (3) and (4)):

$$
H_{b o s}=\left(i \partial_{\mu}-e A_{\mu}(x)\right)\left(i \partial^{\mu}-e A^{\mu}(x)\right)
$$

Thus, omitting all terms originating from the fermion part in (6), i.e. putting $L_{3}=0$ in (12), (15) and $S_{c l}^{f e r}=0$ in (21), we come to the following expression

$$
\begin{aligned}
& \left\langle x\left|U_{b o s}(\tau)\right| x\right\rangle=-\frac{i}{16 \pi^{2} \tau^{2}} \frac{\left(e \tau K_{-}\right)\left(e \tau K_{+}\right)}{\sin \left(e \tau K_{-}\right) \sinh \left(e \tau K_{+}\right)} \\
& \times\left[1-\frac{i}{8} e F_{\nu \lambda, \mu \kappa} \sum_{j, l} C^{V}\left(f_{j}, f_{l}\right)\left(A_{(j)}^{\nu \lambda} A_{(l)}^{\mu \kappa}+2 A_{(j)}^{\nu \mu} A_{(l)}^{\lambda \kappa}\right)+\frac{i}{18} e^{2} F_{\nu \lambda, \mu} F_{\sigma \kappa, \rho}\right. \\
& \times \sum_{j, l, k}\left(C_{1}^{V V}\left(f_{j}, f_{l}, f_{k}\right)\left(A_{(j)}^{\nu \lambda} A_{(l)}^{\kappa \sigma} A_{(k)}^{\mu \rho}+A_{(j)}^{\nu \mu} A_{(l)}^{\kappa \rho} A_{(k)}^{\lambda \sigma}+2 A_{(j)}^{\nu \lambda} A_{(l)}^{\kappa \rho} A_{(k)}^{\mu \sigma}\right)\right. \\
& +C_{2}^{V V}\left(f_{j}, f_{l}, f_{k}\right)\left(A_{(j)}^{\nu \sigma} A_{(l)}^{\kappa \lambda} A_{(k)}^{\mu \rho}+A_{(j)}^{\nu \rho} A_{(l)}^{\kappa \mu} A_{(k)}^{\lambda \sigma}+2 A_{(j)}^{\nu \sigma} A_{(l)}^{\kappa \mu} A_{(k)}^{\lambda \rho}\right) \\
& +2 C_{3}^{V V}\left(f_{j}, f_{l}, f_{k}\right)\left(A_{(j)}^{\nu \lambda} A_{(l)}^{\kappa \mu} A_{(k)}^{\sigma \rho}+A_{(j)}^{\kappa \rho} A_{(l)}^{\nu \sigma} A_{(k)}^{\lambda \mu}\right)+C_{4}^{V V}\left(f_{j}, f_{l}, f_{k}\right) A_{(j)}^{\nu \kappa} A_{(l)}^{\lambda \mu} A_{(k)}^{\sigma \rho} \\
& \left.\left.+C_{5}^{V V}\left(f_{j}, f_{l}, f_{k}\right) A_{(j)}^{\nu \kappa}\left(A_{(l)}^{\lambda \sigma} A_{(k)}^{\mu \rho}+A_{(l)}^{\lambda \rho} A_{(k)}^{\mu \sigma}\right)\right)\right] .
\end{aligned}
$$


The coefficients used here are the same as those that were used in (35). In the special case of the electromagnetic field of the form (38) we obtain

$$
\left\langle x\left|U_{b o s}(\tau)\right| x\right\rangle_{d e r}=\frac{1}{(8 \pi)^{2} \tau} \frac{\partial_{\mu} \Phi \partial^{\mu} \Phi}{\Phi^{2}}\left(\frac{3(\omega \operatorname{coth} \omega-1)}{\sinh ^{2} \omega}+\omega \operatorname{coth} \omega-2\right) \frac{\omega}{\sinh \omega} .
$$

To end this section, let us write down also the explicit expressions for the effective Lagrangians in the case of a space-time-inhomogeneous magnetic field, which is assumed to be small in comparison with the fermion mass scale. As follows from our formulae (3), (35), (40) and (42), after performing the integration over the proper-time parameter, the corresponding expressions for two-derivative terms are:

$$
\begin{aligned}
\mathcal{L}_{\text {der }}^{\text {fer }} & =-\frac{e^{2} \partial_{\mu} B \partial^{\mu} B}{120 \pi^{2} m^{2}}\left(1-\frac{20}{21}\left(\frac{e B}{m^{2}}\right)^{2}+\ldots\right), \\
\mathcal{L}_{\text {der }}^{\text {bos }} & =-\frac{e^{2} \partial_{\mu} B \partial^{\mu} B}{960 \pi^{2} m^{2}}\left(1-\frac{53}{21}\left(\frac{e B}{m^{2}}\right)^{2}+\ldots\right) .
\end{aligned}
$$

These results for four dimensional QED can be compared with those in the case of three dimensional QED in the presence of a space-inhomogeneous magnetic field. For $\mathrm{QED}_{3}$ (under the same conditions) it was found [3]

$$
\begin{aligned}
\mathcal{L}_{\text {der }}^{\text {fer }} & =\frac{\partial_{i} B \partial_{i} B}{(e B)^{3}} \frac{m^{3}}{60 \pi}\left(\frac{e B}{m^{3}}\right)^{3}+\ldots \\
\mathcal{L}_{\text {der }}^{\text {bos }} & =\frac{\partial_{i} B \partial_{i} B}{(e B)^{3}} \frac{m^{3}}{240 \pi}\left(\frac{e B}{m^{3}}\right)^{3}+\ldots
\end{aligned}
$$

These are precisely the expressions that led the authors of the paper [3] to the conclusion that the appearance of inhomogeneous magnetic fields is accompanied by a decrease of the vacuum energy. The latter would imply a vacuum instability which tends to generate inhomogeneous magnetic field in the vacuum state. However, the question of vacuum stability is inaccessible in a derivative expansion, and in fact the infinite sum of terms with derivatives drives a system towards a system with uniform magnetic field 29].

In the case of $\mathrm{QED}_{4}$, a space-inhomogeneous magnetic field may lead to decreasing the vacuum energy (recall that we use Minkowski metric) as well. However, as in the three dimensional case, it may be only an artifact of the derivative expansion approximation. 


\section{Conclusions}

The main result of our paper is the derivative expansion of the effective action for both the fermion (see formulae (3) and (35)) and the scalar (see formulae (40) and (42)) electodynamics in $3+1$ dimensions. Note that our results, in contrast to those obtained in the paper [2], are derived in an explicit form for the most general electromagnetic field configuration.

As for the application of the derivative expansion derived in the paper, it could be useful in extending the dynamical chiral symmetry breaking in $\mathrm{QED}_{4}$ [5, 6] by a magnetic field to the case where inhomogeneities are present 2 . This would allow contact with the GSI heavy-ion experiments [31] where strong inhomogeneous electromagnetic fields play an important role.

Another application could be connected with the existence of very strong, inhomogeneous fields during the electroweak phase transition in the early Universe [32 which might essentially change the character of that phase transition.

In conclusion we note that the technique used in the present paper can be applied to generate the derivative expansion around covariantly constant non-abelian and gravitational fields. The first term in such an expansion has already been calculated by Savvidi [33] with a result generalizing Schwinger's old result [1] to the non-abelian gauge-field. Recently, such a calculation was extended to the case of nonvanishing, covariantly constant, Riemann curvature by Avramidi [34].

\section{Acknowledgements}

We wish to acknowledge D.G.C.McKeon from the University of Western Ontario (London, Canada) for fruitful discussions. V.P.G. is grateful to the members of the Institute for Theoretical Physics (the University of Groningen, The Netherlands), especially D.Atkinson, for their hospitality during his stay at Groningen University where the final version of the paper was written. V.P.G. would like to thank the Stiching FOM (Fundamenteel Onderzoek der Materie), financially supported by the Nederlandse Organisatie voor Wetenschappelijk Onderzoek, for its support.

The work was supported by the grant INTAS-93-2058 "East-West network in constrained dynamical systems" and in part (for I.A.Sh.) by the International

\footnotetext{
${ }^{2}$ In connection with this see the recent paper [30], generalizing some results of Ref. [5, 6] to the case of an inhomogeneous magnetic field.
} 
Soros Science Education Program (ISSEP) through grant PSU052143.

\section{Appendix}

Here we give the functions that were used in (35):

$$
\begin{aligned}
& C^{W}(\alpha, \beta)=\tau^{2} \tanh (\alpha \tau) H(\beta \tau), \\
& C^{V}(\alpha, \beta)=\alpha \tau^{3} H(\alpha \tau) H(\beta \tau)-\frac{\alpha \tau}{\beta^{2}-\alpha^{2}}[H(\beta \tau)-H(\alpha \tau)], \\
& C_{1}^{W W}(\alpha, \beta, \gamma)=\frac{\tau^{3}}{8} \tanh (\alpha \tau) \tanh (\beta \tau) H(\gamma \tau) \\
& C_{2}^{W W}(\alpha, \beta, \gamma)=\frac{\tau^{2}}{4}[\tanh (\alpha \tau)+\tanh (\beta \tau)]\left(\frac{H(\alpha \tau+\beta \tau)-H(\gamma \tau)}{\alpha+\beta-\gamma}-\frac{H(\gamma \tau)}{\alpha+\beta}\right), \\
& C_{1}^{V W}(\alpha, \beta, \gamma)=-\frac{\tau^{3}}{4} \tanh (\alpha \tau)\left(\beta \tau H(\beta \tau) H(\gamma \tau)-\frac{H(\beta \tau)-H(\gamma \tau)}{\tau(\beta+\gamma)}\right) \\
& C_{2}^{V W}(\alpha, \beta, \gamma)=\frac{\tau^{2} \beta \tanh (\alpha \tau)}{2\left(\beta^{2}-\gamma^{2}\right)}[H(\beta \tau)-H(\gamma \tau)] \\
& C_{1}^{V V}(\alpha, \beta, \gamma)=\frac{\tau^{5} \alpha \beta}{2} H(\alpha \tau) H(\beta \tau) H(\gamma \tau)-\frac{\tau^{3} \alpha H(\alpha \tau)}{2(\beta-\gamma)}(H(\beta \tau)-H(\gamma \tau)) \\
& -\frac{\tau^{3} \beta H(\beta \tau)}{2(\alpha+\gamma)}(H(\alpha \tau)-H(\gamma \tau))-\frac{\tau}{2} \frac{H(\alpha \tau)}{(\alpha+\gamma)(\alpha+\beta)} \\
& -\frac{\tau}{2} \frac{H(\beta \tau)}{(\alpha+\beta)(\beta-\gamma)}+\frac{\tau}{2} \frac{H(\gamma \tau)}{(\alpha+\gamma)(\beta-\gamma)}, \\
& C_{2}^{V V}(\alpha, \beta, \gamma)=-\frac{\tau^{3} \alpha \beta H(\alpha \tau) H(\beta \tau)}{2(\alpha-\beta)(\alpha-\beta+\gamma)}+\frac{\tau^{3}[2(\alpha-\beta)+\gamma]}{2(\alpha-\beta)(\alpha-\beta+\gamma)} \\
& \times H(\gamma \tau)[\beta H(\beta \tau)-\alpha H(\alpha \tau)] \\
& +\frac{\alpha \tau}{2} H(\alpha \tau)\left(\frac{2(\beta+\gamma)}{\left(\alpha^{2}-\beta^{2}\right)\left(\alpha^{2}-\gamma^{2}\right)}-\frac{2 \alpha-\beta+\gamma}{(\alpha-\beta)^{2}(\alpha+\gamma)(\alpha-\beta+\gamma)}\right) \\
& +\frac{\beta \tau}{2} H(\beta \tau)\left(\frac{2(\gamma-\alpha)}{\left(\alpha^{2}-\beta^{2}\right)\left(\beta^{2}-\gamma^{2}\right)}+\frac{2 \beta-\alpha-\gamma}{(\alpha-\beta)^{2}(\beta-\gamma)(\alpha-\beta+\gamma)}\right) \\
& +\frac{\tau}{2} H(\gamma \tau)\left(\frac{2\left(\alpha \beta+\gamma^{2}\right)}{\left(\alpha^{2}-\gamma^{2}\right)\left(\beta^{2}-\gamma^{2}\right)}-\frac{\gamma}{(\alpha+\gamma)(\beta-\gamma)(\alpha-\beta+\gamma)}\right) \\
& +\frac{\tau}{2(\alpha-\beta)(\alpha-\beta+\gamma)} \text {, }
\end{aligned}
$$




$$
\begin{aligned}
& C_{3}^{V V}(\alpha, \beta, \gamma)=-\frac{\tau^{3} \alpha \beta H(\alpha \tau)}{\beta^{2}-\gamma^{2}}[H(\beta \tau)-H(\gamma \tau)]+\frac{\alpha \tau H(\alpha \tau)}{(\alpha-\beta)\left(\alpha^{2}-\gamma^{2}\right)} \\
& -\frac{\tau}{\beta^{2}-\gamma^{2}}\left(\frac{\beta}{\alpha-\beta} H(\beta \tau)-\frac{\alpha \beta+\gamma^{2}}{\alpha^{2}-\gamma^{2}} H(\gamma \tau)\right) \\
& C_{4}^{V V}(\alpha, \beta, \gamma)=-\tau^{3} H(\beta \tau) H(\gamma \tau)-\frac{2 \tau \alpha^{2} H(\alpha \tau)}{\left(\alpha^{2}-\beta^{2}\right)\left(\alpha^{2}-\gamma^{2}\right)}+\frac{\tau\left(\alpha^{2}+\beta^{2}\right) H(\beta \tau)}{\left(\alpha^{2}-\beta^{2}\right)\left(\beta^{2}-\gamma^{2}\right)} \\
& +\frac{\tau\left(\alpha^{2}+\gamma^{2}\right) H(\beta \tau)}{\left(\alpha^{2}-\gamma^{2}\right)\left(\gamma^{2}-\beta^{2}\right)} \\
& C_{5}^{V V}(\alpha, \beta, \gamma)=\frac{\tau^{3} \alpha}{2}\left(\frac{\alpha}{(\alpha+\beta)(\alpha+\beta+\gamma)}+\frac{1}{\alpha+\gamma}\right) H(\alpha \tau) H(\beta \tau) \\
& +\frac{\tau^{3} \alpha}{2}\left(\frac{\alpha}{(\alpha+\gamma)(\alpha+\beta+\gamma)}+\frac{1}{\alpha+\beta}\right) H(\alpha \tau) H(\gamma \tau) \\
& +\frac{\tau[H(\beta \tau)-H(\gamma \tau)]}{\beta^{2}-\gamma^{2}}+\frac{\tau^{3}}{2}\left(\frac{\beta \gamma(\beta+\gamma)}{\alpha+\beta+\gamma}-2 \alpha^{2}\right) \frac{H(\beta \tau) H(\gamma \tau)}{(\alpha+\beta)(\alpha+\gamma)} \\
& +\frac{\alpha \tau H(\alpha \tau)\left(2+\frac{\alpha+\beta}{\alpha+\gamma}+\frac{\alpha+\gamma}{\alpha+\beta}\right)}{2(\alpha+\beta)(\alpha+\gamma)(\alpha+\beta+\gamma)}+\frac{\tau}{2}\left(\frac{H(\gamma \tau)+H(\beta \tau)}{(\alpha+\beta)(\alpha+\gamma)}\right. \\
& +\frac{H(\gamma \tau)-H(\beta \tau)}{(\alpha+\beta)(\beta-\gamma)}+\frac{H(\gamma \tau)-H(\beta \tau)}{(\alpha+\gamma)(\beta-\gamma)}+\frac{\gamma H(\gamma \tau)}{(\alpha+\gamma)^{2}(\alpha+\beta+\gamma)} \\
& \left.+\frac{\beta H(\beta \tau)}{(\alpha+\beta)^{2}(\alpha+\beta+\gamma)}-\frac{1}{(\alpha+\beta)(\alpha+\beta+\gamma)}-\frac{1}{(\alpha+\gamma)(\alpha+\beta+\gamma)}\right) \text {. }
\end{aligned}
$$

Here we used the notation

$$
H(x)=\frac{x \operatorname{coth}(x)-1}{x^{2}} .
$$




\section{References}

[1] J.Schwinger, Phys. Rev. 82 (1951) 664.

[2] H.W.Lee, P.Y.Pac and H.K.Shin, Phys. Rev. D40 (1989) 4202.

[3] D.Cangemi, E.D’Hoker and G.Dunne, Phys. Rev. D51 (1995) R2513.

[4] C.Martin and D.Vautherin, Phys. Rev. D38 (1988) 3593.

[5] V.P.Gusynin, V.A.Miransky and I.A.Shovkovy, Phys. Rev. Lett. 73, (1994) 3499.

[6] V.P.Gusynin, V.A.Miransky and I.A.Shovkovy, Kiev preprint ITP-95-02E, hep-ph/9501304 (to appear in Phys. Rev. D).

[7] D.G.Caldi and S.Vafaeisefat, Phys. Lett. B287, (1992) 185; Phys. Lett. B356 (1995) 386; A.Chodos, Proper-Time Methods in the Presence of NonConstant Background Fields, preprint YCTP-P14-94, to appear in Proceedings of the first Gürsey Memorial Conference.

[8] M.P.Fry, Phys. Rev. D51 (1995) 810.

[9] S.Minakshisundaram and A.Pleijel, Can. J. Math. 1 (1949) 242.

[10] B.S.DeWitt, Dynamical Theory of Groups and Fields, (Gordon and Breach, NY, 1965).

[11] A.O.Barvinsky and G.A.Vilkovisky, Phys. Reports 119 (1985) 1.

[12] G.A.Vilkovisky, preprint CERN-TH 6392/92.

[13] I.G.Avramidi, Nucl. Phys. B355 (1991) 712.

[14] A.O.Barvinsky and G.A.Vilkovisky, Nucl. Phys. B382 (1987) 163; Nucl. Phys. B333 (1990) 512.

[15] A.O.Barvinsky, Yu.V.Gusev, G.A.Vilkovisky and V.V.Zhytnikov, J. Math. Phys. 35 (1994) 3543.

[16] P.B.Gilkey, J. Diff. Geom. 10 (1975) 601.

[17] S.Fulling and G.Kennedy, Trans. Amer. Math. Soc. 310 (1988) 583.

[18] V.P.Gusynin, Phys. Lett. B225 (1989) 233; Nucl. Phys. B333 (1990) 296.

[19] V.P.Gusynin, E.V.Gorbar and V.V.Romankov, Nucl. Phys. B362 (1991) 449. 
[20] V.P.Gusynin and V.A.Kushnir, Yad. Fiz. 51 (1990) 587; Class. Quant. Grav. 8 (1991) 279.

[21] E.S.Fradkin, Nucl. Phys. 76 (1966) 588; E.S.Fradkin and D.M.Gitman, Phys. Rev. D44 (1991) 3230.

[22] V.Yu.Fainberg and A.V.Marshakov, Nucl. Phys. B306 (1988) 659.

[23] J.W. van Holten, Propagators and Path Integrals. Preprint NIKHEF-H/95050, hep-th/9508136.

[24] M.J.Srtassler, Nucl. Phys. B385 (1992) 145.

[25] D.G.C.McKeon, Can. J. Phys. 71 (1993) 334.

[26] M.G.Schmidt and C.Schubert, Phys. Lett. B318 (1993) 438.

[27] V.A.Fock,Sov. Phys. 12 (1937) 404; P.Pascual and R.Tarrach, QCD renormalization for the practitioner, Lecture Notes in Physics 194 (Springer, Berlin, 1984) pp.168-184.

[28] I.A.Batalin, A.E.Shabad, JETP 60 (1971) 894.

[29] D.Cangemi, E.D'Hoker and G.Dunne, Effective Energy for $Q E D_{2+1}$ with Semi-Localized Static Magnetic Fields: A Solvable Model. Preprint UCLA/95/TEP/21, hep-th/9506085.

[30] R.R.Parwani, On Chiral Symmetry Breaking by External Magnetic Fields in $\mathrm{QED}_{3}$, hep-th/9504020.

[31] P.Salabura et al., Phys. Lett. B245 (1990) 153, and references therein.

[32] J.Ambjørn and P.Olesen, Nucl. Phys. B330 (1990) 193.

[33] G.K.Savvidi, Phys. Lett. B71 (1977) 133.

[34] I.G.Avramidi, A New Algebraic Approach for Calculating the Heat Kernel in Quantum Gravity, preprint of University of Greifswald, EMA-MAT-1994-5. 